\title{
INGEIS RADIOCARBON LABORATORY DATES III
}

\author{
ROBERTO CORDERO, N. G. GUIDA and J. L. NOGUEIRA
}

INGEIS, Pabellón INGEIS, Ciudad Universitaria, 1428 Buenos Aires, Argentina

\section{INTRODUCTION}

The following list presents results of dating of 55 paleoenvironmental and archaeological samples from Argentina, processed between 1984 and 1986 by M. A. Albero and F. Angiolini. Procedures for sample pretreatment, counting, statistical analysis and age calculation were essentially the same as previously described by Albero and Angiolini (1985). Results are reported as conventional ${ }^{14} \mathrm{C}$ dates in years before AD 1950. They are corrected for isotopic fractionation.

\section{ACKNOWLEDGMENTS}

We thank the Consejo Nacional de Investigaciones Científicas y Técnicas for financial support and E. Linares for his collaboration. We also wish to thank the Stable Isotope Laboratory staff for $\delta^{13} \mathrm{C}$ analysis and Estela Ducós for her help in the translation.

\section{PALEOENVIRONMENTAL SAMPLES}

\section{La Poma Series}

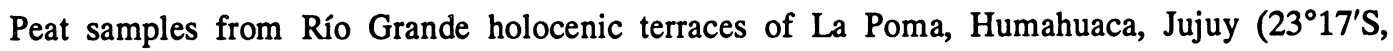
$\left.65^{\circ} 42^{\prime} \mathrm{W}\right), 3600 \mathrm{~m}$ asl. Collected and submitted 1983 by J. Fernández.

\section{AC-691}

Depth $4.30 \mathrm{~m}$

$3280 \pm 90$

$\delta^{13} C=-25.9 \pm 0.2 \%$

AC-692

Depth $4.50 \mathrm{~m}$

$3460 \pm 120$

$\delta^{13} C=-26.0 \pm 0.2 \%$

Comment (J.F.): Dates related to leaf-shaped stemless points. The paleoenvironmental interpretation and chronology have been published in Coleman (1973) and Fernández (1973).

\section{Guayatayoc Series}

Peat samples from Guayatayoc stream, Guayatayoc, Rinconada Jujuy $\left(22^{\circ} 18^{\prime} \mathrm{S}, 66^{\circ} 02^{\prime} \mathrm{W}\right), 3500 \mathrm{~m}$ asl. Collected and submitted 1983 by J. Fernández.
AC-0733
$9170 \pm 170$
Depth $1.82 \mathrm{~m}$
$\delta^{13} C=-27.4 \pm 0.2 \%$
AC-0734
$10,390 \pm 190$
Depth $1.97 \mathrm{~m}$
$\delta^{13} C=-26.5 \pm 0.2 \%$ 。

Comment (J.F.): Data belonging to this same profile were published in Albero and Angiolini (1985: 333). Human occupation, ca. $2800 \mathrm{yr} \mathrm{BP}$, is indicated by quartzitic artifacts. 


\section{Leuto Caballo Series}

Peat, marl and other carbonatic sediments from terraces of the Bordo Leuto Caballo stream $\left(37^{\circ} 05^{\prime} \mathrm{S}, 70^{\circ} 15^{\prime} \mathrm{W}\right), 1670 \mathrm{~m}$ asl, Chos Malal, Neuquén. Collected and submitted 1983 by J. Fernández. All samples belong to peat layers.

AC-0980

Depth $9.67 \mathrm{~m}$

AC-0981

Depth $8.57 \mathrm{~m}$

AC-0979

Depth $7.67 \mathrm{~m}$

AC-0983

Depth $7.52 \mathrm{~m}$

AC-1048

Depth $7.32 \mathrm{~m}$
$12,280 \pm 160$

$\delta^{13} \mathrm{C}=-25.0 \pm 0.2 \% 0$

$11,940 \pm 160$

$\delta^{13} C=-27.9 \pm 0.2 \%$

$11,300 \pm 160$

$\delta^{13} \mathrm{C}=-25.0 \pm 0.2 \%$

$11,070 \pm 140$

$\delta^{13} C=-25.0 \pm 0.2 \%$

$10,180 \pm 130$

$\delta^{13} C=-7.0 \pm 0.2 \%$

Comment (J.F.): Dates are related to a Late Pleistocenic marshy environment with megafaunal remains (Megatherium, Hippidion and Paleolama); see Panarello and Fernández (1992).

\section{Barro Negro Series}

Peat, marl and collagen of several profiles from Barro Negro $\left(23^{\circ} \mathrm{S}, 65^{\circ} 37^{\prime} \mathrm{W}\right), 3.820 \mathrm{~m}$ asl, Humahuaca, Jujuy. Collected and submitted $1980-1986$ by J. Fernández. All samples belong to peat layers.

A) Quebrada Linda Profile (Site A)

AC-0748

Depth $0.20 \mathrm{~m}$

AC-0740

Depth $1.42 \mathrm{~m}$

AC-0738

Depth $2.10 \mathrm{~m}$

AC-0739

Depth $2.80 \mathrm{~m}$

AC-0735

Depth $8.40 \mathrm{~m}$

B) Quebrada de Las Piedras Profile

AC-0747

Depth $1.17 \mathrm{~m}$

AC-0673

Depth $1.60 \mathrm{~m}$

AC-0682

Depth $1.70 \mathrm{~m}$
$510 \pm 70$

$\delta^{13} C=-25.3 \pm 0.2 \%$

$940 \pm 80$

$\delta^{13} C=-27.6 \pm 0.2 \%$ 。

$1270 \pm 80$

$\delta^{13} C=-25.4 \pm 0.2 \%$ 。

$1690 \pm 80$

$\delta^{13} C=-27.6 \pm 0.2 \%$

$12,530 \pm 160$

$\delta^{13} \mathrm{C}=-26.8 \pm 0.2 \% 0$

$1000 \pm 80$

$\delta^{13} C=-24.0 \pm 0.2 \%$

$1140 \pm 80$

$\delta^{13} C=-23.7 \pm 0.2 \%$ 。

$1680 \pm 120$

$\delta^{13} C=-25.0 \pm 0.2 \%$ 
AC-0680

Depth $1.90 \mathrm{~m}$

AC-0746

Depth $2.12 \mathrm{~m}$

AC-0745

Basal depth $5.82 \mathrm{~m}$

\section{AC-0744}

Basal depth $6.77 \mathrm{~m}$

Comment: Marl and collagen samples were found in basal peat layer

AC-0974

Marl 2. depth $5.90 \mathrm{~m}$

AC-0975

Marl 4. depth $6.20 \mathrm{~m}$

AC-0976

Marl 5. depth $6.80 \mathrm{~m}$

AC-0969 I

Collagen from Hippidion bone.

AC-0969 II

Collagen from Hippidion bone.

C) Zanjon Largo Profile

AC-0742

Peaty silt with Lymnaea, depth $4.56 \mathrm{~m}$

AC-0743

Peaty silt with fragmented camel bones and lithic objects, depth $5.08 \mathrm{~m}$
$2890 \pm 110$

$\delta^{13} C=-24.3 \pm 0.2 \%$

$3470 \pm 90$

$\delta^{13} C=-27.5 \pm 0.2 \%$

$10,200 \pm 140$

$\delta^{13} C=-25.0 \pm 0.2 \%$

$12,300 \pm 170$

$\delta^{13} \mathrm{C}=-28.7 \pm 0.2 \%$

Comment (J.F.): Important superior Pleistocenic and Holocenic chronostratigraphic record with archaeological contents, fossil animal remains and paleoenvironmental indicators. Published in Alberdi et al. (1986); Fernández et al. (1991); Fernández (1983-1985).

\section{ARCHAEOLOGICAL SAMPLES}

\section{Haichol Series}

Charcoal samples from Haichol cave $\left(38^{\circ} 35^{\prime} \mathrm{S}, 70^{\circ} 40^{\prime} \mathrm{W}\right) 1050 \mathrm{~m}$ asl. Pehuenches, Neuquén. Collected and submitted 1979-1981 by J. Fernández.

AC-0896

Grid 13B2. depth $0.40-0.50 \mathrm{~m}$

AC-0897

Grid 9B1, depth $0.50-0.60 \mathrm{~m}$

AC-0898

Grid 14B1, depth $0.50-0.60 \mathrm{~m}$
$9870 \pm 220$

$\delta^{13} \mathrm{C}=-4.0 \pm 0.2 \%$

$13,400 \pm 400$

$11,600 \pm 160$

$\delta^{13} C=-3.8 \pm 0.2 \%$

$9120 \pm 130$

$\delta^{13} C=-18.0 \pm 0.2 \% 0$

$11,500 \pm 400$

$\delta^{13} C=-18.0 \pm 0.2 \% 0$ 
AC-0899

$2290 \pm 120$

Grid 16B2, depth $0.50-0.60 \mathrm{~m}$

$\delta^{13} C=-22.6 \pm 0.2 \% 0$

AC-0900

$2130 \pm 110$

Grid 11B2, depth $0.60-0.70 \mathrm{~m}$

$\delta^{13} C=-22.9 \pm 0.2 \%$

AC-0901

$2440 \pm 100$

Grid 17B2, 0.80-0.90 m

$\delta^{13} C=-21.9 \pm 0.2 \% 0$

Comment (J.F.): Dates belonging to Late Preceramic occupations (2440-2130 yr BP) and Early Pottery occupations (1830-1250 yr BP). Published in Fernández (1988-1990).

Vizcayachoc Peat

Peat from lower part of Vizcachayoc peat bog, north of Miyuyoc $\left(22^{\circ} 55^{\prime} \mathrm{S}, 65^{\circ} 20^{\prime} \mathrm{W}\right) 4000 \mathrm{~m}$ asl, Humahuaca, Jujuy. Collected and submitted 1983 by J. Fernández.

AC-0982

$9540 \pm 170$

$\delta^{13} \mathrm{C}=-21.2 \pm 0.2 \%$

Comment (J.F.): Lower part of peat bed $7 \mathrm{~m}$ width, on western slope of Cordillera Oriental.

Pluma De Pato

Eggshell of Pterocnemia pennata (choique) from Pluma del Pato, Añelo, Neuquén (38 ${ }^{\circ} 10^{\prime} \mathrm{S}, 69$ $\left.5^{\prime} \mathrm{W}\right) 250 \mathrm{~m}$ asl. Collected and submitted 1979 by J. Fernández.

AC-1049

$1700 \pm 90$

$\delta^{13} \mathrm{C}=-8.0 \pm 0.2 \%$

Comment (J.F.): Associated with ceramics and triangular stemless projectile points with concave base, made of obsidian.

\section{Ojo De Agua}

Interbedded wood at $2.10 \mathrm{~m}$ depth in a small fluvial terrace in Ojo de Agua, near Susques $\left(23^{\circ} 23^{\prime} \mathrm{S}\right.$, $66^{\circ} 22^{\prime} \mathrm{W}, 3700 \mathrm{~m}$ asl. Collected and submitted 1983 by J. Fernández.

AC-1053

Wood

$\delta^{13} C=-22.6 \pm 0.2 \%$

Calcium carbonate biostromal accretion from Pastos Chicos $\left(23^{\circ} 45^{\prime} \mathrm{S}, 6^{\circ} 23^{\prime} \mathrm{W}\right) 3900 \mathrm{~m}$ asl. Collected and submitted 1983 by J. Fernández.

AC-1056

$16,250 \pm 290$

$\delta^{13} \mathrm{C}=+8.8 \pm 0.2 \%$

Comment (J.F.): Stromatolite (oncolite) from ancient Pastos Chicos stream flood terrace.

\section{Anillaco Series}

Charcoal samples from Faldeos del Anillaco, La Rioja ( $28^{\circ} 46^{\prime} \mathrm{S}, 66^{\circ} 57^{\prime} \mathrm{W}$.) $1500 \mathrm{~m}$ asl. Collected and submitted 1984 by R. Raffino.

AC-919

$1160 \pm 120$

Depth $0.60 \mathrm{~m}$ 
AC-920

$1200 \pm 120$

Depth $0.70 \mathrm{~m}$

AC-921

$2080 \pm 120$

Depth $0.80 \mathrm{~m}$

AC-922

$1330 \pm 120$

Depth $0.90 \mathrm{~m}$

AC-923

Depth $1.00 \mathrm{~m}$

$1330 \pm 120$

AC-924

Depth $1.10 \mathrm{~m}$

$\delta^{13} C=-22.9 \%$

AC-925

$1150 \pm 140$

Depth $1.20 \mathrm{~m}$

$\delta^{13} C=-21.0 \%$

AC-926

$1440 \pm 120$

Depth $1.30 \mathrm{~m}$

$\delta^{13} C=-23.8 \%$

$1270 \pm 120$

\section{La Huerta Series}

Bone and charcoal samples from La Huerta, Jujuy, $\left(23^{\circ} 20^{\prime} \mathrm{S}, 65^{\circ} 20^{\prime} \mathrm{W}\right) 2700 \mathrm{~m}$ asl. Collected and submitted 1985 by R. Raffino and published by Raffino et al.(1993).

AC-960

$$
\begin{array}{r}
480 \pm 100 \\
\delta^{13} C=-16.5 \% 0
\end{array}
$$

Bone collagen, between 0.22 and $0.32 \mathrm{~m}$

AC-1069

$540 \pm 90$

Charcoal, depth $0.12 \mathrm{~m}$

\section{Valle De La Ciénaga Series}

Charcoal samples from La Ciénaga valley, Tucumán $\left(26^{\circ} 49^{\prime} \mathrm{S}, 65^{\circ} 39^{\prime} \mathrm{W}\right) 2700 \mathrm{~m}$ asl. Collected and submitted 1984 by B. Cremonte.

AC- 720

Depth $1.40 \mathrm{~m}$

$1560 \pm 80$

AC-721

Depth $0.80 \mathrm{~m}$

$\delta^{13} C=$ est. 0

$1240 \pm 80$

$\delta^{13} \mathrm{C}=-24.80 \%$

Dates related to large settlements of early potter and agricultural societies from the northwest of Argentina, especially with those of Tafí valley. Published by Cremonte (1988).

\section{Puesto El Rodeo Series}

Vegetal remains and charcoal samples from La Magdalena estancia, Río Pinturas area, Santa Cruz, $\left(46^{\circ} 53^{\prime} \mathrm{S}, 70^{\circ} 27^{\prime} \mathrm{W}\right) 240 \mathrm{~m}$ asl. Collected and submitted 1985 by C. Gradin and A. Aguerre.
AC-943
Depth $0.47 \mathrm{~m}$
$1380 \pm 90$
$\delta^{13} \mathrm{C}=-22.1 \%$


AC-1075

Depth $0.90 \mathrm{~m}$

Comment: Dates correspond to vegetal remains found over a bedded burial place containing three skeletons. The first date is related to the Rio Pinturas IV cultural level and the second one to the Rio Pinturas IIb-III cultural level.

\section{Puesto Giles Series}

Charcoal samples from Puesto Giles Site, Casa de Piedra, La Pampa ( $\left.38^{\circ} 11^{\prime} \mathrm{S}, 67^{\circ} 13^{\prime} \mathrm{W}\right) 250 \mathrm{~m}$ asl. Collected and submitted 1983 by C. Gradin and A. Aguerre.

AC-728

Depth $0.53 \mathrm{~m}$

AC-731

Depth $0.40 \mathrm{~m}$
$700 \pm 100$

$\delta^{13} \mathrm{C}=-21.3 \%$

$320 \pm 120$

$\delta^{13} C=-21.7 \%$

Comment: Dates related to a late human occupation of hunter-gatherers who used ceramics (under investigation).

\section{El Cuy Series}

Charcoal sample from El Cuy, Cañadón Santa Victoria, Río Negro $\left(40^{\circ} \mathrm{S}, 68^{\circ} \mathrm{W}\right) 400$ asl. Collected and submitted 1986 by C. Gradin and A. Aguerre.

AC-1074

$420 \pm 110$

Depth between 0.05-0.20 m

$\delta^{13} C=-21.2 \%$

Comment: Date related to tool remains made of bone and a stemmed point, with small patagoniensetype fins. Investigation was stopped at prospecting stage.

\section{REFERENCES}

Alberdi, M. T., Fernandez, J., Menegaz, A. N. and Prado, J. L. 1986 Hippidion owen 1869 (Mammalia, Perissodactyla) en sedimentos del Pleistoceno tardío de la localidad de Barro Negro (Jujuy, Argentina). Estudios Geológicos 42: 487-493.

Albero, M. C., and Angiolini, F. 1985 INGEIS Radiocarbon Laboratory dates II. Radiocarbon 27(2B): 314 337.

Coleman, D. 1973. Illinois State Geological Survey radiocarbon dates IV. Radiocarbon 15(1): 314-337.

Cremonte, M. B. 1988 Comentario acerca de fechados radiocarbónicos del sitio El Pedregal (Qda. La Ciénaga, Tucumán, Argentina). Revista Chungará 20: 57-59.

Fernandez, J. 1973 Primeros fechados radicarbónicos de formaciones holocenas de la Puna, portadoras de materiales arqueológicos. Actas del Quinto Congreso Geologico Argentino, Vol. 3. Buenos Aires, Argentina: 223-226.

1983-1985 Cronología y paleoambientes del intervalo 12.550-520 años A.P. (Pleistoceno-Holoceno) de la Puna jujeña. Anales de Arqueologia y Etnología 3840: $29-42$.
1988-1989 Ocupaciones alfareras $(2.860 \pm 160$ años A.P.) en la Cueva de Cristóbal, Puna de Jujuy, Argentina. Relaciones de la Sociedad Argentina de Antropología 17(2): 139-182.

1988-1990. La cueva de Haichol. Arqueología de los pinares cordilleranos del Neuquén. Anales de Arqueología y Etnologia 43-45: 1-738.

Fernandez, J., Markgraf, V., Panarello, H. O., Albero, M., Angiolini, F. E., Valencio, S. and Arriaga, M. 1991 Late Pleistocene / Early Holocene environments and climates, fauna and human occupation in the Argentine altiplano. Geoarchaeology 6(3): 251-272.

Panarello, H. O., and Fernandez, J. (ms.) 1992 First paleotemperature record in arid northwest Patagonia (Neuquén, Argentina) during the late Pleistocene-Holocene transition (12.300-10180 yr B.P.) derived from pallustrine shells (Lymnnaea) and marl ${ }^{18} \mathrm{O}$ analyses. Report submited to the International Atomic Energy Agency. Vienna. Austria.

Raffino, R. A. 1993 Inka. Arqueología, Historia y Urbanismo del Altiplano Andino. Buenos Aires, Ed. Corregidor: $318 \mathrm{p}$. 